\title{
Experiencia familiar frente a la muerte materna
}

\author{
Impact of maternal mortality in the family
}

Experiência familiar diante da morte materna

\author{
Ivonne Romero-Guzmán ${ }^{1 *}$ \\ Dennys Isabel Muñoz-Monteroza ${ }^{2}$ \\ Lucelly Benitez-Cheij ${ }^{3}$
}

\begin{abstract}
Autor de correspondencia
${ }^{1 *}$ Enfermera. Magister en Enfermería. Docente Universidad del Sinú Elías Bechara Zainúm. Montería, Colombia. Correo: ivonnerogu@hotmail.com (iD

2 Enfermera. Magister en Salud Pública. Docente Universidad del Sinú Elías Bechara Zainúm. Montería, Colombia. Correo: dennysisabelm83@gmail.com (D)

${ }^{3}$ Enfermera. Especialista en Epidemiologia. Docente Universidad del Sinú Elías Bechara Zainúm. Montería, Colombia. Correo: lbenitezcheij@hotmail.com (iD)
\end{abstract}

(C) Universidad Francisco de Paula Santander. Este es un artículo bajo la licencia CC-BY-NC-ND (c)

\section{Resumen}

Introducción: La mortalidad materna constituye un problema de salud pública y un indicador de desarrollo y sostenibilidad de los países. Además, es un problema social que genera gran impacto a nivel familiar, en el aspecto económico, en el legal y el educativo, produciéndose muchas veces separación de los integrantes de la familia. Objetivo: Describir la experiencia de la familia frente a la muerte materna. Materiales y Métodos: Estudio de tipo etnográfico y enfoque cualitativo, circunscrito en el marco de la dinámica del método de Etnoenfemería de Leininger. Se entrevistaron 6 familias de la Ciudad de Montería, en donde se presentó un evento de mortalidad materna. Resultados: Se identificaron tres temas principales: "la madre mantiene fuertemente los lazos afectivos en la familia", "experiencia difícil y dolorosa de las familias durante el proceso de agonía de su ser querido" y "la pérdida de la madre genera cambios en la dinámica familiar, sufrimiento emocional y disminuye los recursos económicos". Conclusión: Se puede concluir, que la experiencia familiar frente a la muerte materna incluye cambios en la dinámica familiar, en los roles de cuidadores y en la desintegración familiar; del mismo modo, afecta el aspecto económico, el rendimiento escolar e impacta fuertemente en al ámbito emocional de la familia.

Palabras claves: Muerte materna; familia; impacto psicosocial; mortalidad materna; enfermería.

\section{Abstract}

Introduction: Maternal mortality constitutes a public health problem and it is an indicator of development and sustainability for a country. Also, it is a social problem that generates great impact on the family level, and in the economic, legal and educational aspects, often producing the separation of the family members. Objective: Describe the impact of maternal mortality in the family. Materials and methods: Ethnographic research and qualitative focus circumscribed within the framework of the dynamics from the Ethnonursing method of Leininger. Six families from the city of Monteria, Colombia, were interviewed during an event about maternal mortality. Results: Three main subjects were identified: "the mother maintains strong affective ties in the family", "a hard and painful experience for the family during the dying

Recibido: 9 enero 2020 Aprobado: 22 abril 2020

Para citar este artículo/ To reference this article/ Para citar este artigo/

Romero-Guzmán Ivonne; Muñoz-Monteroza Dennys Isabel; Benitez-Cheij Lucelly. Experiencia familiar frente a la muerte materna. Rev. cienc. cuidad. 2020; 17(2): 44-52. https://doi.org/10.22463/17949831.1887 
moments of their loved one" and "the loss of a mother generates changes in the dynamics of the family, emotional suffering and it reduces the income". Conclusion: It can be concluded that the impact of maternal mortality in the family includes changes in the dynamics of the family, the roles of caregivers and the breakup of the family; similarly, it affects the economic aspect, the academic performance and the emotional field of family.

Keywords: Death of the mother; family; psychosocial impact; maternal mortality; nursing.

\begin{abstract}
Resumo
Introdução: A mortalidade materna representa um problema de saúde pública, sendo também um indicador de desenvolvimento e sustentabilidade dos países. Além disso, gera impacto familiar, social, econômico, legal e educativo, produzindo a separação das famílias. Objetivo: Descrever a experiência das famílias diante da morte materna. Materiais e métodos: Estudo etnográfico, qualitativo empregando o método de etno-enfermagem de Leininger. Entrevistaram-se 6 famílias da cidade de Montería onde apresentou-se um óbito materno. Resultados: Identificaram-se três temas principais: "a mãe mantém estreitamente os laços afetivos na família"; "experiência difícil e dolorida das famílias durante o processo da agonia do seu ser querido"; "a perda da mãe gera mudanças na dinâmica familiar: sofrimento emocional e redução dos recursos econômicos". Conclusão: Concluise que a experiência familiar diante da morte materna inclui mudanças na dinâmica familiar, os papeis de cuidadores e a desintegração familiar; igualmente, afeta a economia familiar, o rendimento acadêmico e impacta severamente o equilíbrio emocional da família.
\end{abstract}

Palavras-chave: Morte materna; família; impacto psicossocial; mortalidade materna; enfermagem.

\section{Introducción}

La mortalidad materna constituye un problema de salud pública y un indicador del desarrollo y sostenibilidad de los países (1); además, refleja el fracaso de los sistemas sanitarios en la protección de la salud de la mujer (2). Según la Organización Mundial de la Salud (OMS) se evidencia que alrededor de 830 mujeres mueren diariamente por complicaciones relacionadas con el embarazo o el parto. Este fenómeno es alarmante en países en vía de desarrollo, en comunidades residentes en zonas rurales y mujeres adolescentes, como se refleja en el año 2015, en donde se reportaron 303000 muertes por esta causa; es decir, que existe una razón de mortalidad materna ratio mayor en países en desarrollo de 239 por 100000 nacidos vivos, versus 12 por 100 000, razón de mortalidad materna ratio presentada en los países desarrollados (3).

Así mismo, en América Latina en las últimas décadas se han dado cambios sociopolíticos y económicos importantes, que han permitido disminuir los altos índices de mortalidad y morbilidad materna, apareciendo en las agendas de los distintos países estrategias para enfrentar este flagelo (4). Según datos aportados por la Organización Panamericana de la salud, en América Latina la razón de mortalidad materna fue de 58,2 por cada cien mil nacidos vivos en el año 2017 (5), lo que demuestra que aún existen muchos aspectos por mejorar desde las políticas públicas, para afrontar este evento.

En Colombia, según el Análisis de la Situación en Salud de 2018 emitido por el Ministerio de Salud y Protección Social, en el periodo comprendido entre los años 2005 y 2017, la razón de mortalidad materna se redujo de 70,1 a 51,01 muertes maternas por cada 100.000 nacidos vivos; sin embargo, aún hay que realizar esfuerzos que contribuyan en reducir la razón nacional de mortalidad materna a menos de 32,0 por cada 100.000 nacidos vivos (6).

Por su parte, el Departamento de Córdoba no es ajeno a este fenómeno, el comportamiento de la mortalidad materna se ha destacado por ser uno de los más altos del país. Durante el 2016, la razón de mortalidad materna a los 42 días fue de 65,59 casos en 100.000 nacidos vivos, siendo superior a la media nacional (7). En 2017 representó un 5,4\% de los casos a nivel nacional (8). En el año 2018 la razón fue de 72,1 y en 2019 hasta la semana epidemiológica 27 fue de 34 (9).

Se debe precisar que las causas de mortalidad materna están relacionadas con factores obstétricos asociados a complicaciones del embarazo, parto o puerperio, mal manejo médico y comorbilidades que alteran el curso del embarazo (10-12). Igualmente, existen determinantes sociales que influyen en la ocurrencia de este 
evento como: el nivel socioeconómico bajo, la raza, baja escolaridad y el vivir en zonas dispersas; lo que supone, que estos factores inciden directamente en el acceso a los servicios de salud (13-14). Varios estudios han demostrado que al disminuir estas inequidades se produce una reducción significativa del número de muertes maternas (15).

La muerte materna, además, constituye un problema social que genera gran impacto a nivel familiar, afectando el aspecto económico, el legal y el educativo; es por eso, que muchas veces se produce separación de los integrantes de la familia, como es el caso de los hijos que quedan al cuidado de un familiar. La ocurrencia de una muerte materna marca el futuro de los hijos y de toda la familia, ya que ellas constituyen en muchos casos el motor de los hogares (16-17).

En este sentido, diversas investigaciones han reportado que la muerte materna expone a los hijos huérfanos a situaciones que afectan su bienestar, afrontando la pérdida de la madre y los cambios que esto conlleva. Desde esta perspectiva, aumenta el riesgo de morbilidades a nivel físico y mental, bajo rendimiento y deserción escolar; circunstancias que pueden afectarlos hasta la adultez y que por tanto refuerzan el ciclo de pobreza en estas familias (18).

En efecto, esta temática se ha abordado en diferentes estudios e informes estadísticos; sin embargo, existe escasa información publicada sobre el impacto de la muerte materna en la familia. Por tanto, es necesario conocer la forma en que las familias afrontan la pérdida y el duelo, identificando los aspectos que más afectan a los huérfanos, para así crear herramientas que contribuyan a brindar un cuidado integral que propenda a una mejor calidad de vida de dichas familias.

\section{Objetivo}

Describir la experiencia de la familia frente a la muerte materna.

\section{Materiales y métodos}

Estudio de tipo etnográfico y enfoque cualitativo, circunscrito en el marco de la dinámica del método de Etnoenfemería de Leininger, el cual permite el estudio y la clasificación sistemática de las creencias, valores, prácticas, significados y experiencias en la vida de las personas relacionadas con el cuidado, la salud y bienestar del ser humano (19).
La muestra fue determinada con base en la información necesaria para el estudio. En este sentido, se tuvo en cuenta el último trimestre del año 2019. Se trabajó con seis familias de la Ciudad de Montería en las cuales se presentó un evento de mortalidad materna. Así mismo, se seleccionaron como informantes clave, 18 familiares cercanos a la familia materna. Se alcanzó el punto de saturación cuando no se encontró nueva información o la misma fue redundante; por consiguiente, en el muestreo se siguió el proceso que señalan Salamanca y Martín (20).

De acuerdo con el método seleccionado y a las propiedades del estudio, la obtención de la información se realizó mediante el uso de técnicas como la entrevista a profundidad y el diario de campo. Las entrevistas a profundidad realizadas en el hogar incluyeron seis preguntas abiertas de inicio, que permitieron a los participantes responder con sus propias palabras, y expresar abiertamente sus experiencias y sentimientos, permitiendo al investigador profundizar en la temática, formular nuevas preguntas y obtener la información necesaria. En el diario de campo se registraron las percepciones y apreciaciones de las investigadoras, obteniendo así una visión completa de la experiencia vivida por los participantes. Posteriormente, se procedió a la revisión de las cintas de grabación y del diario de campo, para el respectivo análisis de información acorde a las fases de análisis propuestas por Leininger (21).

Siguiendo el método Etnoenfermero de Leininger, se desarrollaron cuatro fases de análisis de la información:

En la primera fase se reunió, describió, clasificó y analizó la información; también, se incluyeron las observaciones, la identificación del significado del contexto, las interpretaciones y la identificación de símbolos.

En la segunda fase se identificaron y categorizaron descriptores y componentes. Los descriptores son estudiados para determinar sus semejanzas y diferencias y los componentes recurrentes para entender su significado.

En la tercera fase se buscaron patrones y el análisis contextual. La información se examinó para descubrir la saturación de ideas y patrones recurrentes de significados, expresiones y formas similares y diferentes.

En la cuarta fase se identificaron los temas de mayor importancia presentes en el discurso de las familias que se vieron afectadas, a consecuencia de la muerte materna. 
Las cuatro fases dieron por resultado la identificación de 171 significados de contexto, procesadas analíticamente en 18 descriptores, 8 patrones y 3 temas centrales. Se incluyeron los criterios de rigor metodológico, de credibilidad, de auditabilidad y de adecuabilidad (22).

Respecto a las consideraciones éticas, este estudio se rigió por la Resolución 8430 de 1993 y los Principios Éticos de Autonomía, Beneficencia y Privacidad. Las familias que aceptaron participar fueron informadas sobre los objetivos del estudio, y aprobaron su participación mediante la firma de un consentimiento informado. Se veló por resguardar la confidencialidad de los datos de cada uno de los participantes, mediante la codificación de cada caso. El estudio contó con la aprobación del Comité de Ética en Investigación de la Facultad Ciencias de la Salud, de la Universidad del Sinú Elías Bechara Zainum.

\section{Resultados}

El análisis mediante el método etnoenfermero facilitó la identificación de tres temas principales: la madre mantiene fuertemente los lazos afectivos en la familia; la experiencia es difícil y dolorosa para las familias durante el proceso de agonía de su ser querido; $y$, la pérdida de la madre genera cambios en la dinámica familiar, sufrimiento emocional y disminuye los recursos económicos.

\section{La madre mantiene fuertemente los lazos afectivos en la familia.}

En este tema se lograron identificar dos patrones recurrentes: funcionalidad familiar y relaciones afectivas. En este sentido, las familias expresan cambios en la funcionalidad de la familia:

"Con mi mamá antes se hacía lo que ella decía, con mi papá no, porque él es muy relajado, a él no le gusta estar mandando ni discutiendo, pero mi mamá sí" CODIGO F2C2.

“...pues antes había problemas, pero como estaba uno con la mamá no se sienten casi, pero ahora si se sienten más los problemas, las discusiones familiares y todo eso" CODIGO F5P12.

Así mismo comentan la experiencia que tuvieron al lado de la madre, cómo se mantenía la relación afectiva que existía entre los integrantes de la familia:
"Antes éramos muy afectivos muy unidos y nos ha afectado siempre, porque ella era la que estaba aquí en casa, ella me ayudaba con mis hijos, después que ella murió todo se ha volteado, nada es igual me hace mucha falta" CODIGO F3C3.

De igual manera, las familias expresan que la pérdida de su ser querido generó en ellos grandes cambios reflejados en la variación de los roles de los integrantes de la familia; es decir, que otros miembros adoptaron otros roles con el fin de equilibrar y tratar de mantener la funcionalidad de la familia; los cambios más notorios se ven reflejados en el cuidador:

\section{"Mi papá me va a pasar para que yo quede como tutora de mis hermanas" CODIGO F5C5.}

Además, las familias manifiestan que la funcionalidad de la familia cambió de una forma u otra a raíz de la ausencia materna. De ahí, que dicha ausencia afecte tanto a las familias, presentándose en algunos casos la desintegración familiar; esto es, que por falta de un miembro en la familia, principalmente por la pérdida de la madre, se presente el abandono del hogar, de los hijos o del padre:

“...el papá de los niños vivía acá con nosotras, pero cuando ella falleció él se fue, pero le da la mensualidad de ellos" CODIGO F4C4.

"pero cuando murió ella, la hijita mayor de ella se fue a vivir con los abuelitos, para allá, para la tierra de la mamá" CODIGO F6C6.

\section{Experiencia difícil y dolorosa de las familias durante el proceso de agonía de su ser querido.}

En este tema se observaron los siguientes patrones recurrentes: las familias perciben déficit en el trato humanizado del personal de salud; desconocimiento y dificultad en la comunicación, para determinar el estado de salud y bienestar de su ser querido; necesidad de ayuda económica; carencia de apoyo y acompañamiento emocional que requirieron las familias durante el proceso de enfermedad; hospitalización de las madres; $y$, sentimientos negativos de la familia hacia el personal de salud.

A este respecto, las familias manifiestan haber vivido una experiencia difícil y dolorosa, tanto por el proceso de enfermedad y muerte de la madre, como por los inconvenientes que tuvieron con el personal de salud durante este tiempo, al sentir que en algunas ocasiones observa- 
ISSN-PRINT

1794-9831

E-ISSN 2322-7028

Vol. 17 No. 2

May - Ago 2020

Cúcuta, Colombia ban indiferencia y deshumanización por miembros del personal de salud:

"En la noche ya ella comenzó a sudar y que se sentía mal y yo fui donde la enfermera y le pedi el favor que le fuera a tomar la presión y ella me dijo que todavía no porque todavía no era el turno o no sé" CODIGO F5C5.

... ella se me desmayó y yo corrí y llamé a una enfermera y la enfermera me dijo que ella lo que estaba haciendo era teatro, que ella no estaba tan mal, sino que estaba haciendo era teatro" CODIGO F6C6.

Igualmente, se evidencia falta de información que debe ser transmitida a las familias sobre: los tratamientos, procedimientos, evolución, entre otros; ya que, al momento de preguntar sobre el proceso de enfermedad de la madre, los entrevistados expresaron no tener conocimientos claros al respecto:

"Nunca me explicaron nada, en sí de que fue lo que murió ella" CODIGO F1C1.

"Solamente me dijeron que la iban a operar, yo no sabía de qué la iban a operar, nunca me dijeron nada" CODIGO F4C4.

El dolor emocional y el sufrimiento que les invadía al ver como la vida de su ser querido se desvanecía ante ellos:

"Uno como mamá pues a uno le duele eso, de todas maneras, uno puede tener los hijos que tenga, pero si le hace falta uno le hace falta" CODIGO F4P8. "Solloza, pone su mirada en sus manos" CODIGO ODCF4.

"A mi se me derrumbó todo, es muy doloroso" CODIGO F2C3. "Se tapa la boca por un instante, aun con su mirada desviada responde" CODIGO ODCF2.

Al respecto, también se evidencia la ayuda y apoyo que les brindan los familiares ante la pérdida reciente de su ser querido, como son las ayudas económicas, lo emocional y el acompañamiento psicológico:

"Ahora mis tíos nos colaboran, los abuelos y todos nos han estado apoyando, cosa que no hacían cuando estaba viva ella" CODIGO F5C5.

"En una de esas nos tocó llamar un tío de nosotros, para que nos facilitara dinero y unas amigas de mi mamá, con esa plata nosotros pagamos el sepelio y todo" CODIGO F2C2.
La pérdida de la madre genera cambios en la dinámica familiar, sufrimiento emocional y disminuye los recursos económicos.

En este tema se identificaron los siguientes patrones recurrentes: las familias experimentan dolor, sufrimiento y tristeza al afrontar la muerte de la madre, así como necesidades económicas.

En las familias, perder a su ser querido tuvo repercusiones en varios aspectos de sus vidas, especialmente en la dinámica familiar. En relación con esto, se generaron cambios notables, más si se tiene en cuenta, que la familia ya no está completa, debido a la falta de un integrante , como lo es la ausencia de la madre. También se generaron separaciones físicas, debido a que los hijos o el padre abandonaban el hogar que tenían conformado con el apoyo materno:

“... antes viviamos las cuatro y mi padrastro, cuando ella murió mi padrastro se fue y mis hermanas quedaron con mis abuelos, porque yo vivo acá con mi suegra y mi novio" CODIGO F5C5.

En las familias donde quedaron huérfanos, también se generaron cambios en ellos a nivel educativo, puesto que al ocurrir la muerte de la madre el rendimiento escolar empezó a decaer, mientras otros se vieron en la necesidad de abandonar los estudios:

"ella estaba estudiando y cuando murió la mamá empezó a irle mal en el colegio que hasta perdió el año" CODIGO F4C4.

Hay que destacar, que igualmente el ámbito económico se ve afectado, puesto que la madre dentro de la familia generaba ingresos económicos y de cierta forma su falta representa menos ingresos, por lo que las familias se vieron en la obligación de trabajar aún más, para suplir sus necesidades:

"Yo estaba estudiando y me sali para poder trabajar, mi hermana se encarga de las cosas de la casa, a cualquiera le puede pasar, que la mamá se le muera y uno ilusionado con la mamá, pero ajá, pasan esas cosas inesperadas" CODIGO F2C2.

\section{Discusión}

El rol de la madre en la sociedad es fundamental, culturalmente es la encargada en mayor medida de la crianza de los hijos, siendo el pilar en la estructura del hogar (22). Por lo que una muerte materna desencadena en 
una serie de situaciones en la familia, tales como desequilibrio emocional y económico. Estos factores provocan en los individuos que la conforman un giro en sus vidas, puesto que una madre representa un fundamento vital en la familia debido a su rol de cuidadora, educadora y protectora.

A través de este estudio cualitativo se comprobó mediante las experiencias relatadas en las entrevistas, que el impacto de una muerte materna en la familia se refleja de manera negativa, en todos los aspectos de la vida de los individuos que la conforman; esto debido a que la madre siempre será un pilar vital en el desarrollo de la dinámica familiar y su desaparición definitivamente se hará notar.

Un evento de mortalidad materna causa en la pareja, los hijos y la familia cercana, sentimientos de soledad y vacío emocional. En este sentido, se ha descrito que en sucesos de duelo familiar son los niños los más afectados, por la fragilidad que presentan. Es por eso, que los niños huérfanos tienen un menor desarrollo en la integración de las defensas y en el cognitivo, generando modificaciones en su mundo interno; como consecuencia atraviesan por fases de turbulencia afectiva, que si no se abordan adecuadamente desde el plano familiar y de apoyo interdisciplinar, pueden desencadenar en desapego, depresión e incluso en psicopatologías (24).

Aunado a la situación anterior, uno de los cambios observados es la desintegración familiar, en donde los huérfanos fueron delegados a otros familiares, lo que concuerda con lo observado por Rodríguez et al., quienes expresan que los viudos acuden a sus suegras o cuñadas para el cuidado de los hijos para poder cumplir con su rol de proveedores económicos (25). Esta situación puede provocar en los hijos sufrimiento y desequilibrio emocional, dificultándoles la adaptación al nuevo entorno y al desarrollo integral, especialmente en las áreas de lenguaje, personal y social (26).

Otra de las secuelas familiares que se observaron posterior al fallecimiento de la madre, la hija o la esposa, están relacionadas con el nivel económico. Tal es el caso de los gastos relacionados con el funeral, esto a nivel inmediato; a largo plazo, se observa disminución de los ingresos económicos, especialmente en los hogares donde la madre era activa laboralmente. $\mathrm{Al}$ respecto Kes et al. (27), reportaron que hay un mayor gasto relacionado con el acceso y uso de la atención médica en los hogares donde se produjo una muerte materna, frente a aquellos que tuvieron un embarazo y un parto sanos. Así mismo, se observaron grandes costos asociados a los funerales, razón por la cual los hogares se vieron obligados a liquidar activos o a realizar prestamos, así como a redistribuir las responsabilidades laborales para compensar las contribuciones, frente a la pérdida de las mujeres fallecidas.

En lo referente al aspecto económico, las familias expresaron la importancia del apoyo de los otros familiares al enfrentar este tipo de consecuencias, señalando que los familiares directos han asumido la responsabilidad de apoyo económico para el hogar; esta situación se replica en diferentes culturas en donde al ocurrir una muerte materna, otros miembros de la familia inmediata proporcionan alimentos y otros recursos; sin embargo, esta situación en algunos casos puede producir tensiones entre los miembros de la familia extensa, cuando estas expectativas de ayuda no son cumplidas (28) .

De acuerdo con lo señalado por Pande (29) los hijos de las madres fallecidas presentan deserción escolar o disminuyen en su rendimiento, en muchos casos por asumir tareas del hogar o responsabilidades laborales, producto del deterioro económico del mismo; esta situación es similar a lo expresado por las familias del estudio, donde se reflejó deserción escolar y bajo rendimiento en los huérfanos.

En otro orden de ideas, se debe precisar que las bajas condiciones económicas, un deficiente control prenatal, una precaria atención en salud y un mal diagnóstico son los detonantes para dar lugar a la muerte de una madre (30). En este sentido, se observó inconformidad en el servicio brindado por el personal de salud desde la perspectiva familiar, resaltando la deshumanización e indiferencia. Estos resultados concuerdan con lo reportado por estudios anteriores, en donde los familiares experimentaron sentimientos de abandono y desigualdad en la atención sanitaria, enmarcada en la deshumanización (31).

A este respecto, es necesario replantear la atención que se brinda y el trato humanizado hacia la gestante y su familia, de tal forma que permita generar en ellos satisfacción con la atención prestada. Así mismo, es fundamental reforzar los esfuerzos desde los centros de atención primaria para brindar un mejor seguimiento desde la atención preconcepcional, el control prenatal y la preparación para el parto; elementos que están presentes en este nivel donde se concentran las condiciones necesarias para el abordaje integral de los riesgos detectados (32).

En este aspecto Gonzaga et al. (33), indican que la atención adecuada de la mujer embarazada se tradu- 
ce en la vigilancia prenatal, mediante el seguimiento y control periódico que permitan la identificación y el diagnóstico oportuno de los riesgos, lo que redundará en una reducción directa de la mortalidad materna.

En concordancia con los planteamientos anteriores, las familias también expresaron falta de información y desconocimiento sobre las complicaciones que presentaron las madres y las causas de muerte; resultados similares a lo evidenciado en otras investigaciones, en donde las sobrevivientes de morbilidad materna extrema percibieron vulneración de sus derechos, al no ser informadas sobre su estado de salud, las causas de las complicaciones o decidir sobre los procedimientos en dichos momentos (34-35). En relación con lo anterior, se ha identificado que los elementos organizacionales y culturales son los que influyen mayormente en la calidad de la atención a estas pacientes. Al respecto, los más relevantes corresponden a los protocolos de atención, por lo que es indispensable, que por parte de las instituciones de salud se adopten protocolos o guías en la atención de pacientes obstétricas. Por tanto, deben estar basados en la evidencia científica y orientados a responder los requerimientos de esta población y su familia, tales como la información adecuada en los eventos de morbimortalidad. Desde esta perspectiva, no es posible hablar de calidad de la atención obstétrica sin la implementación y la adherencia de dichos protocolos (36).

Por todo lo anterior, se concluye que la muerte de una madre puede afectar a la familia a nivel emocional, a su funcionamiento e incluso al ámbito económico y educativo, por lo que resulta imperativo, no solo conocer las vivencias familiares después de la muerte materna sino, que además, hay que implementar políticas y estrategias que permitan el seguimiento adecuado, para dar respuesta a las necesidades emocionales y sociales que estas familias presentan.

\section{Conclusiones}

Es posible concluir, que la muerte materna es una situación que afecta a la familia, en donde al sentir la ausencia de la madre, se evidencia el importante rol que cumplía. Al mismo tiempo, se percibieron cambios en la dinámica familiar, en los roles de los cuidadores, en el aspecto económico y en el emocional, entre otros.

Es preciso resaltar que el hecho de hacer frente a la muerte de la madre por parte de las familias se convierte en un evento muy difícil; sin embargo, intentan adaptarse y superar el suceso desafortunado, en donde las redes de apoyo cumplen un rol fundamental.

De otra parte, la responsabilidad económica, es un aspecto que influye en el modo de manejar la situación por parte de la familia, especialmente cuando la madre sobrellevaba la responsabilidad económica; razón por la cual, al faltar ella, los miembros de la familia se ven obligados a redireccionar sus vidas en el ámbito laboral, para sustentar de forma efectiva a todos los miembros.

Cabe destacar, que la experiencia con el equipo de salud por parte de los familiares, no fue agradable de acuerdo con sus afirmaciones, convirtiendo estas narraciones en un reto para todos los profesionales de la salud, en cuanto a la humanización de la atención, no solo con la madre en este caso, sino también con la familia que la acompaña. Por esta razón, es necesaria la implementación de métodos que permitan una mejor dinámica, con la que se ofrezca una intervención óptima, en donde el rol de la enfermería debe ser fundamental en el acompañamiento continuo de la gestante, puérpera y su familia, brindando una atención humanizada que se traduzca en el arte de cuidar.

Para este estudio fue de suma importancia la teoría del cuidado cultural de Leininger, puesto que permitió a las investigadoras conocer las experiencias de las familias, teniendo en cuenta la cultura en la que están inmersas, su sentir más profundo, sus valores, sus creencias y sus necesidades. Atendiendo a la consideración anterior, es importante fortalecer este tipo de investigación desde el enfoque cultural en enfermería, que permita brindar un cuidado integral a los individuos y a su familia.

\section{Conflicto de intereses}

Los autores declaran que para el desarrollo del presente estudio no existió conflicto de intereses. 


\section{Referencias bibliográficas}

1. Programa de las Naciones Unidas para el Desarrollo. Objetivos del Desarrollo Sostenible [Internet]. Washington D.C.: Nueva York: PNUD; 2015. Disponible en: http:/www.undp.org/content/undp/es/ home/sustainable-development-goals.html

2. Bess-Constantén S, Martínez-Morales MA, Fernández-Viera MR, Mazorra-Ramos V, Alonso-Alomá I, López-Nistal LM, et al. Calidad de las estadísticas de mortalidad materna en Cuba. Rev Panam Salud Publica. 2018;42: D47. doi: https://doi.org/10.26633/RPSP.2018.47

3. Organización Mundial de la Salud. Mortalidad Materna. Centro de prensa. [Internet] Septiembre, 2019. Disponible en: https://www.who.int/es/news-room/fact-sheets/detail/maternal-mortality

4. Centro Latinoamericano de Perinatología, Salud de la Mujer y Reproductiva. Reducción mortalidad materna en 11 países de la región. [Internet] 2015. Disponible en: http://www.paho.org/clap/index. php?option $=$ com_content $\&$ view $=$ article $\&$ id $=220$ :reduccion-mortalidad-materna-en-11-paises-de-laregion\&Itemid $=354 \&$ showall $=1 \&$ lang $=$ es

5. Pan American Health Organization. Health Situation in the Americas. Core Indicators 2018. PAHO: Washington, D.C.; 2018.

6. Ministerio de Salud y Protección Social. Análisis de Situación de Salud (ASIS) Colombia, 2018. [Internet] Junio 2019. Disponible en: https://www.minsalud.gov.co/sites/rid/Lists/BibliotecaDigital/ RIDE/VS/ED/PSP/asis-colombia-2018.pdf

7. Programa de las Naciones Unidas para el Desarrollo PNUD Colombia. Córdoba, retos y desafíos para el Desarrollo Sostenible. 2019

8. Ministerio de Salud y Protección Social. Informe del evento Mortalidad Materna. Instituto Nacional de Salud. Grupo Maternidad Segura. [Internet] 2017. Disponible en: https://www.ins.gov.co/buscadoreventos/Informesdeevento/MORTALIDAD\%20MATERNA\%202017.pdf

9. Departamento de Córdoba. Secretaria de Desarrollo de la Salud. Sivigila 2018-2019.

10. Soni-Trinidad C, Gutiérrez-Mateos A, Santa Rosa-Moreno FJ, Reyes-Aguilar A. Morbilidad y mortalidad materna y factores de riesgo asociados con una urgencia obstétrica. Ginecol Obstet Mex 2015; 83 (02)

11. Márquez-Gómez MA, Gómez-Díaz GM. Vigilancia del uso de medicamentos en el embarazo en el municipio de Los Palmitos, Sucre, Colombia: una contribución para la prevención y reducción de la mortalidad materna y perinatal. Nova 26 de octubre de 2017;15(28):115-24. https://doi.org/10.22490/24629448.2086

12. Carton J, de Paula SF, de Andrade GB, Rangel RF, Ventura J, de Siqueira CH. Maternal Mortality Profile: An Integrative Literature Review. Revista de Pesquisa, Cuidado é Fundamental Online 2019 Apr; 11(3):816-822.

13. Sanhueza A, Roldán JC, Ríos-Quituizaca P, Acuña MC, Espinosa I. Social inequalities in maternal mortality among the provinces of Ecuador. Revista Panamericana de Salud Pública 2017; 41.

14. Mera-Mamián AY, Alzate-Sánchez RA. Mortalidad materna en el departamento de Cauca, un estudio en el régimen subsidiado. Revista de la Facultad Nacional de Salud Pública 2019; 37(3):64-73.

15. Mantin MA, Zavattiero C. Evolución de la mortalidad materna en Argentina y Paraguay entre 1990 y 2015; políticas públicas, desigualdades y desafíos. Sociedad y Economía 2019(38):21-44.

16. Salazar E, Buitrago C, Molina F, Arango Alzate C. Tendencia de la mortalidad por causas externas en mujeres gestantes o puérperas y su relación con factores socioeconómicos en Colombia, 1998-2010. Rev Panam Salud Pública. 2015; 37(4/5):225-231.

17. Lozano-Avendaño L, Bohórquez-Ortiz AZ, Zambrano-Plata GE. Implicaciones familiares y sociales de la muerte materna. Rev Univ. Salud. 2016; 18(2):364-372

18. Miller S, Belizán JM. The true cost of maternal death: individual tragedy impacts family, community and nations. Reprod Health. 2015; 12:56. https://doi.org/10.1186/s12978-015-0046-3

19. Houle B, Clark SJ, Kahn K. Tollman S, Yamin A. The impacts of maternal mortality and cause of death 
ISSN-PRINT

$1794-9831$

E-ISSN 2322-7028

Vol. 17 No. 2

May - Ago 2020

Cúcuta, Colombia on children's risk of dying in rural South Africa: evidence from a population-based surveillance study (1992-2013). Reprod Health. 2015; 12-S7. https://doi.org/10.1186/1742-4755-12-S1-S7

20. Salamanca-Castro AB, Martín-Crespo MC. El muestreo en la investigación cualitativa. Nure Investigación. 2007; 27-07.

21. Leininger M. Transcultural Nursing: Concepts, Theories and Practices. 1978. New York. Ed: John Wiley and Sons. p. 223-239.

22. Castillo E, Vásquez ML. El rigor metodológico en la investigación cualitativa. Colombia Médica, vol. 34, núm. 3, 2003, pp. 164-167 Vázquez Morales V, Ruvalcaba Ledezma JC. La Salud Materna como Indicador de Inequidad en Salud Pública. JONNPR. 2016;1(3):115-122 DOI: 10.19230/jonnpr.2016.1.3.1021

23. Marín-Iral MP, Quintero-Córdoba PA, Rivera-Gómez SC. Influencia de las relaciones familiares en la primera infancia. Poiésis. 2019; 36:164-183. https://doi.org/10.21501/16920945.3196

24. García-Arce I, Bellver-Pérez A. El proceso de duelo: particularidades en la infancia y adolescencia. Psicobioquímica, 2019; 6: 99 -114.

25. Rodríguez-Leal D, Sánchez de Parada B, Dueñas-Granados PE. Experiencia familiar de la muerte maternal. Rev. cienc. cuidad. 2019; 16(1):71-85.

26. Salomón AC. Situación social familiar y de salud de los huérfanos de muertes maternas en trance obstétrico. Hospital Regional "Dr. Ramon Carrillo". Santiago del Estero. Revista de Salud Pública. 2015; 19(1)-59-69. https://doi.org/10.31052/1853.1180.v19.n1.11812

27. Kes A, Ogwang S, Pande RP, Douglas Z, Karuga R, Odhiambo FO, et al. The economic burden of maternal mortality on households: evidence from three sub-counties in rural western Kenya. Reprod Health. 2015; 12(1). https://doi.org/10.1186/1742-4755-12-S1-S3

28. Bazile J, Rigodon J, Berman L, Boulanger VM, Maistrellis E, et al. Intergenerational impacts of maternal mortality: Qualitative findings from rural Malawi. Reprod Health. 2015; 12(1). https://doi. org/10.1186/1742-4755-12-S1-S1

29. Pande RP, Ogwang S, Karuga R, Rajan R, Kes A, et al. Continuing with “...a heavy heart” - consequences of maternal death in rural Kenya. Reprod Health. 2015; 12(2). https://doi.org/10.1186/17424755-12-S1-S2

30. Kestler E. Política y muerte materna en Guatemala. Rev. méd. (Col. Méd. Cir. Guatem.). 2016; 155:(2):55-58.

31. Villegas N, Cianelli R. Salud de la mujer en el contexto de la salud materna. 2017, Horiz_Enferm, 28, 2, 1-3. DOI UC: 10.7764/Horiz_Enferm.28.2.1

32. Amorim TV, Souza Ívis E de O, Moura MAV, Queiroz ABA, Salimena AMO. Perspectivas de los cuidados de enfermería en el embarazo de alto riesgo: revisión integradora. Enf Global. 2017;16(2):50043. http://dx.doi.org/10.6018/eglobal.16.2.238861

33. Gonzaga-Soriano MR, Zonana-Nacach A, Anzaldo-Campos MC, Olazarán-Gutiérrez A. Atención prenatal y mortalidad materna hospitalaria en Tijuana, Baja California. Salud Pública Mex 2014; 56:32-39.

34. Szulik D, Szwarc L. "Era una bomba de tiempo": el derrotero de la morbilidad materna severa en el Área Metropolitana de Buenos Aires. Salud colectiva. 2015; 11(4):553-564. http://dx.doi.org/10.18294/ sc.2015.795

35. Meaney S, O'Connor L, Lutomski JE, O'Donoghue K, Greene RA. PP32 qualitative analysis of maternal morbidities: the patient's experience. Journal of Epidemiology and Community Health. 2014; 68 :(Suppl1): A60.

36. Semper-González AI, Segredo-Pérez AM, Hernández-Narino A. Calidad de la atención a la morbilidad materna extremadamente grave. Estudio preliminar. Hospital Ginecobstétrico Provincial. Matanzas 2016. Rev. Med. Electrón. 2017; 39(1): 53-60 